PROCEEDINGS OF THE

AMERICAN MATHEMATICAL SOCIETY

Volume 138, Number 6, June 2010, Pages 1989-1994

S 0002-9939(10)10245-7

Article electronically published on February 2, 2010

\title{
DECOMPOSITION OF POLYNOMIALS AND APPROXIMATE ROOTS
}

\author{
ARNAUD BODIN
}

(Communicated by Bernd Ulrich)

\begin{abstract}
We state a kind of Euclidian division theorem: given a polynomial $P(x)$ and a divisor $d$ of the degree of $P$, there exist polynomials $h(x), Q(x), R(x)$ such that $P(x)=h \circ Q(x)+R(x)$, with $\operatorname{deg} h=d$. Under some conditions $h, Q, R$ are unique, and $Q$ is the approximate $d$-root of $P$. Moreover we give an algorithm to compute such a decomposition. We apply these results to decide whether a polynomial in one or several variables is decomposable or not.
\end{abstract}

\section{INTRODUCTION}

Let $A$ be an integral domain (i.e. a unitary commutative ring without zero divisors). Our main result is:

Theorem 1. Let $P \in A[x]$ be a monic polynomial. Let $d \geqslant 2$ be such that $d$ is a divisor of $\operatorname{deg} P$ and $d$ is invertible in $A$. There exist $h, Q, R \in A[x]$ such that

$$
P(x)=h \circ Q(x)+R(x)
$$

with the conditions that

(i) $h, Q$ are monic;

(ii) $\operatorname{deg} h=d$, coeff $\left(h, x^{d-1}\right)=0, \operatorname{deg} R<\operatorname{deg} P-\frac{\operatorname{deg} P}{d}$;

(iii) $R(x)=\sum_{i} r_{i} x^{i}$ with $\left(\operatorname{deg} Q \mid i \Rightarrow r_{i}=0\right)$.

Moreover such $h, Q, R$ are unique.

The previous theorem has a formulation similar to the Euclidian division, but here $Q$ is not given (only its degree is fixed); there is a natural $Q$ (that we will compute, see Corollary 22) associated to $P$ and $d$. Notice also that the decomposition $P(x)=h \circ Q(x)+R(x)$ is not the $Q$-adic decomposition, since the coefficients before the powers $Q^{i}(x)$ belong to $A$ and not to $A[x]$.

Example. Let $P(x)=x^{6}+6 x^{5}+6 x+1 \in \mathbb{Q}[x]$. If $d=6$ we find the following decomposition $P(x)=h \circ Q(x)+R(x)$ with $h(x)=x^{6}-15 x^{4}+40 x^{3}-45 x^{2}+30 x-10$, $Q(x)=x+1$ and $R(x)=0$. If $d=3$ we have $h(x)=x^{3}+65, Q(x)=x^{2}+2 x-4$ and $R(x)=40 x^{3}-90 x$. If $d=2$ we get $h(x)=x^{2}-\frac{725}{4}, Q(x)=x^{3}+3 x^{2}-\frac{9}{2} x+\frac{27}{2}$ and $R(x)=-\frac{405}{4} x^{2}+\frac{255}{2} x$.

Received by the editors March 10, 2009, and, in revised form, October 6, 2009.

2010 Mathematics Subject Classification. Primary 13B25.

Key words and phrases. Decomposable and indecomposable polynomials in one or several variables.

(C)2010 American Mathematical Society Reverts to public domain 28 years from publication 
Theorem 1 will be of special interest when the ring $A$ is itself a polynomial ring. For instance at the end of the paper we give an example of a decomposition of a polynomial in two variables $P(x, y) \in A[x]$ for $A=K[y]$.

The polynomial $Q$ that appears in the decomposition has already been introduced in a rather different context. We denote by $\sqrt[d]{P}$ the approximate $d$-root of $P$. It is the polynomial such that $(\sqrt[d]{P})^{d}$ approximates $P$ in the best way; that is to say, $P-(\sqrt[d]{P})^{d}$ has smallest possible degree. The precise definition will be given in section 2, but we already notice the following:

\section{Corollary 2.}

$$
Q=\sqrt[d]{P}
$$

We apply these results to another situation. Let $A=K$ be a field and let $d \geqslant 2$. $P \in K[x]$ is said to be $d$-decomposable in $K[x]$ if there exist $h, Q \in K[x]$, with $\operatorname{deg} h=d$ such that

$$
P(x)=h \circ Q(x) .
$$

Corollary 3. Let $A=K$ be a field. Suppose that char $K$ does not divide $d . P$ is $d$-decomposable in $K[x]$ if and only if $R=0$ in the decomposition of Theorem 1 .

In particular, if $P$ is $d$-decomposable, then $P=h \circ Q$ with $Q=\sqrt[d]{P}$.

After the first version of this paper, M. Ayad and G. Chèze communicated to us some references so that we can picture a part of the history of the subject. Approximate roots appeared (for $d=2$ ) in some work of E.D. Rainville 9 to find polynomial solutions of some Riccati type differential equations. An approximate root was seen as the polynomial part of the expansion of $P(x)^{\frac{1}{d}}$ into decreasing powers of $x$. The use of approximate roots culminated with S.S. Abhyankar and T.T. Moh, who proved the so-called Abhyankar-Moh-Suzuki theorem in [1] and 2. For the latest subject we refer the reader to an excellent expository article of P. Popescu-Pampu [8]. On the other hand Ritt's decomposition theorems (see [10] for example) have led to several practical algorithms to decompose polynomials in one variable into the form $P(x)=h \circ Q(x)$ : for example D. Kozen and S. Landau in [6] give an algorithm (refined in [5]) that computes a decomposition in polynomial time. Unification of both subjects starts with P.R. Lazov and A.F. Beardon ([7, 3]) for polynomials in one variable over complex numbers: they notice that the polynomial $Q$ is in fact the approximate $d$-root of $P$.

We define approximate roots in section 2 and prove the uniqueness of the decomposition of Theorem 1. Then in section 3 we prove the existence of such a decomposition and give an algorithm to compute it. Finally in section 4 we apply these results to decomposable polynomials in one variable and in section 5 to decomposable polynomials in several variables.

\section{Approximate Roots AND PRoOf of the Uniqueness}

The approximate roots of a polynomial are defined by the following property ([1, 8, Proposition 3.1]).

Proposition 4. Let $P \in A[x]$ be a monic polynomial and let $d \geqslant 2$ be such that $d$ is a divisor of $\operatorname{deg} P$ and $d$ is invertible in $A$. There exists a unique monic polynomial $Q \in A[x]$ such that

$$
\operatorname{deg}\left(P-Q^{d}\right)<\operatorname{deg} P-\frac{\operatorname{deg} P}{d} .
$$


We call $Q$ the approximate $d$-root of $P$ and denote it by $\sqrt[d]{P}$.

Let us recall the proof from 8 .

Proof. We write $P(x)=x^{n}+a_{1} x^{n-1}+a_{2} x^{n-2}+\ldots+a_{n}$ and we search an equation for $Q(x)=x^{\frac{n}{d}}+b_{1} x^{\frac{n}{d}-1}+b_{2} x^{\frac{n}{d}-2}+\cdots+b_{\frac{n}{d}}$. We want $\operatorname{deg}\left(P-Q^{d}\right)<\operatorname{deg} P-\frac{\operatorname{deg} P}{d}$, that is to say, the coefficients of $x^{n}, x^{n-1}, \ldots, x^{n-\frac{n}{d}}$ in $P-Q^{d}$ equal zero. By expanding $Q^{d}$ we get the system of equations

$$
\left\{\begin{array}{l}
a_{1}=d b_{1} \\
a_{2}=d b_{2}+\left(\begin{array}{l}
d \\
2
\end{array}\right) b_{1}^{2} \\
\vdots \\
a_{k}=d b_{k}+\sum_{\substack{i_{1}+2 i_{2}+\cdots+(k-1) i_{k-1}=k \\
i_{1}+i_{2}+\cdots+i_{k-1} \leqslant d}} c_{i_{1} \ldots i_{k-1}} b_{1}^{i_{1}} \cdots b_{k-1}^{i_{k-1}}, \quad 1 \leqslant k \leqslant \frac{n}{d},
\end{array}\right.
$$

where the coefficients $c_{i_{1} \ldots i_{k-1}}$ are the multinomial coefficients defined by the following formula:

$$
c_{i_{1} \ldots i_{k-1}}=\left(\begin{array}{c}
d \\
i_{1}, \ldots, i_{k-1}
\end{array}\right)=\frac{d !}{i_{1} ! \cdots i_{k-1} !\left(d-i_{1}-\cdots-i_{k-1}\right) !} .
$$

With the system $(\mathcal{S})$ being a triangular system, we can inductively compute the $b_{i}$ for $i=1,2, \ldots, \frac{n}{d}: b_{1}=\frac{a_{1}}{d}, b_{2}=\frac{a_{2}-\left(\begin{array}{c}d \\ 2\end{array}\right) b_{1}^{2}}{d}, \ldots$ Hence the system (S) admits one and only one solution $b_{1}, b_{2}, \ldots, b_{\frac{n}{d}}$.

Notice that we need $d$ to be invertible in $A$ to compute $b_{i}$. Moreover $b_{i}$ depends only on the first coefficients $a_{1}, a_{2}, \ldots, a_{\frac{n}{d}}$ of $P$.

Proposition 4 enables us to prove Corollary [2, by condition (iii) of Theorem 1 we know that $\operatorname{deg}\left(P-Q^{d}\right)<\operatorname{deg} P-\frac{\operatorname{deg} P}{d}$ so that $Q$ is the approximate $d$ root of $P$. Another way to compute $\sqrt[d]{P}$ is to use iterations of the Tschirnhausen transformation; see [1] or [8, Proposition 6.3]. We end this section by proving the uniqueness of the decomposition of Theorem 1 .

Proof. $Q$ is the approximate $d$-root of $P$ and, therefore, is unique (see Proposition 4 above). In order to prove the uniqueness of $h$ and $R$, we argue by contradiction. Suppose that $h \circ Q+R=h^{\prime} \circ Q+R^{\prime}$ with $R \neq R^{\prime}$; set $r_{i} x^{i}$ to be the highest monomial of $R(x)-R^{\prime}(x)$. On one hand $x^{i}$ is a monomial of $R$ or $R^{\prime}$, hence $\operatorname{deg} Q \nmid i$ by condition (iii) of Theorem 1. From the equality $\left(h^{\prime}-h\right) \circ Q=R-R^{\prime}$ we deduce that $i=\operatorname{deg}\left(R-R^{\prime}\right)$ is a multiple of $\operatorname{deg} Q$; this yields a contradiction. Therefore $R=R^{\prime}$; hence $h=h^{\prime}$.

\section{Algorithm And proof of the existence}

Here is an algorithm to compute the decomposition of Theorem 1

\section{Algorithm 5.}

- Input. $P \in A[x], d \mid \operatorname{deg} P$.

- Output. $h, Q, R \in A[x]$ such that $P=h \circ Q+R$.

- 1st step. Compute $Q=\sqrt[d]{P}$ by solving the triangular system (S) of Proposition 4, Set $h_{1}(x)=x^{d}, R_{1}(x)=0$. 
- 2nd step. Compute $P_{2}=P-Q^{d}=P-h_{1}(Q)-R_{1}$. Look for its highest monomial $a_{i} x^{i}$. If $\operatorname{deg} Q \mid i$, then set $h_{2}(x)=h_{1}(x)+a_{i} x^{\frac{i}{\operatorname{deg} Q}}, R_{2}=R_{1}$. If $\operatorname{deg} Q \nmid i$, then $R_{2}(x)=R_{1}(x)+a_{i} x^{i}, h_{2}=h_{1}$.

- 3rd step. Set $P_{3}=P-h_{2}(Q)-R_{2}$ and look for its highest monomial $a_{i} x^{i}, \ldots$.

- Iterate the process....

- Final step. $P_{n}=P-h_{n-1}(Q)-R_{n-1}=0$ yields the decomposition $P=h \circ Q+R$ with $h=h_{n-1}$ and $R=R_{n-1}$.

The algorithm terminates because the degree of the $P_{i}$ decreases at each step. It yields a decomposition $P=h \circ Q+R$ that verifies all the conditions of Theorem 1 in the second step of the algorithm and due to Proposition 4 we know that $i<\operatorname{deg} P-$ $\frac{\operatorname{deg} P}{d}$. That implies coeff $\left(h_{2}, x^{d-1}\right)=0$ and $\operatorname{deg} R_{2}<\operatorname{deg} P-\frac{\operatorname{deg} P}{d}$. Therefore at the end coeff $\left(h, x^{d-1}\right)=0$. Of course the algorithm proves the existence of the decomposition in Theorem 1 .

\section{Decomposable polynomials in one variable}

Let $K$ be a field and let $d \geqslant 2 . P \in K[x]$ is said to be $d$-decomposable in $K[x]$ if there exist $h, Q \in K[x]$, with $\operatorname{deg} h=d$ such that

$$
P(x)=h \circ Q(x) .
$$

We refer to [4 for references and recent results on decomposable polynomials in one and several variables.

Proposition 6. Let $A=K$ be a field whose characteristic does not divide $d$. $A$ monic polynomial $P$ is d-decomposable in $K[x]$ if and only if $R=0$ in the decomposition $P=h \circ Q+R$.

In view of Algorithm 5 we also get an algorithm to decide whether a polynomial is decomposable or not and in the positive case we give its decomposition.

Proof. If $R=0$, then $P$ is $d$-decomposable. Conversely if $P$ is $d$-decomposable, then there exist $h, Q \in K[x]$ such that $P=h(Q)$. As $P$ is monic we can suppose $h, Q$ monic. Moreover, up to a linear change of coordinates $x \mapsto x+\alpha$, we can suppose that $\operatorname{coeff}\left(h, x^{d-1}\right)=0$. Therefore $P=h(Q)$ is a decomposition that verifies the conditions of Theorem 1 .

Remark. Let $P(x)=x^{n}+a_{1} x^{n-1}+\cdots+a_{n}$; we first consider $a_{1}, \ldots, a_{n}$ as indeterminates (i.e. $P$ is seen as an element of $\left.K\left(a_{1}, \ldots, a_{n}\right)[x]\right)$. The coefficients of $h(x), Q(x)$ and $R(x)=r_{0} x^{k}+r_{1} x^{k-1}+\cdots+r_{k}$ (computed by Proposition 4, the system ( $(\underline{\mathcal{S}})$ and Algorithm $[5)$ are polynomials in the $a_{i}$, in particular $r_{i}=r_{i}\left(a_{1}, \ldots, a_{n}\right) \in K\left[a_{1}, \ldots, a_{n}\right], i=0, \ldots, k$.

Now we consider $a_{1}^{*}, \ldots, a_{n}^{*} \in K$ as specializations of $a_{1}, \ldots, a_{n}$ and denote by $P^{*}$ the specialization of $P$ at $a_{1}^{*}, \ldots, a_{n}^{*}$. Then, by Proposition 6, $P^{*}$ is $d$-decomposable in $K[x]$ if and only if $r_{i}\left(a_{1}^{*}, \ldots, a_{n}^{*}\right)=0$ for all $i=0, \ldots, k$. It expresses the set of $d$-decomposable monic polynomials of degree $n$ as an affine algebraic variety. We give explicit equations in the following example.

Example. Let $K$ be a field of characteristic different from 2. Let $P(x)=x^{6}+$ $a_{1} x^{5}+a_{2} x^{4}+a_{3} x^{3}+a_{4} x^{2}+a_{5} x+a_{6}$ be a monic polynomial of degree 6 in $K[x]$ (the $a_{i} \in K$ being indeterminates). Let $d=2$. We first look for the approximate 
2-root of $P(x) \cdot \sqrt[2]{P(x)}=Q(x)=x^{3}+b_{1} x^{2}+b_{2} x+b_{3}$. In view of the triangular system (S) we get

$$
b_{1}=\frac{a_{1}}{2}, \quad b_{2}=\frac{a_{2}-b_{1}^{2}}{2}, \quad b_{3}=\frac{a_{3}-2 b_{1} b_{2}}{2} .
$$

Once we have computed $Q$, we get $h(x)=x^{2}+a_{6}-b_{3}^{2}$. Therefore

$$
R(x)=\left(a_{4}-2 b_{1} b_{3}-b_{2}^{2}\right) x^{2}+\left(a_{5}-2 b_{2} b_{3}\right) x .
$$

Now $P(x)$ is 2-decomposable in $K[x]$ if and only if $R(x)=0$ in $K[x]$, that is to say, if and only if $\left(a_{1}, \ldots, a_{6}\right)$ satisfies the polynomial system of equations in $a_{1}, \ldots, a_{5}$ :

$$
\left\{\begin{array}{l}
a_{4}-2 b_{1} b_{3}-b_{2}^{2}=0 \\
a_{5}-2 b_{2} b_{3}=0
\end{array}\right.
$$

\section{Decomposable polynomials in Several variables}

Again $K$ is a field and $d \geqslant 2$. Set $n \geqslant 2 . \quad P \in K\left[x_{1}, \ldots, x_{n}\right]$ is said to be $d$-decomposable in $K\left[x_{1}, \ldots, x_{n}\right]$ if there exist $Q \in K\left[x_{1}, \ldots, x_{n}\right]$ and $h \in K[t]$ with $\operatorname{deg} h=d$ such that

$$
P\left(x_{1}, \ldots, x_{n}\right)=h \circ Q\left(x_{1}, \ldots, x_{n}\right) .
$$

Proposition 7. Let $A=K\left[x_{2}, \ldots, x_{n}\right]$, and let $P \in A\left[x_{1}\right]=K\left[x_{1}, \ldots, x_{n}\right]$ be monic in $x_{1}$. Fix d that divides $\operatorname{deg}_{x_{1}} P$ such that char $K$ does not divide $d . P$ is $d$-decomposable in $K\left[x_{1}, \ldots, x_{n}\right]$ if and only if the decomposition $P=h \circ Q+R$ of Theorem 1 in $A\left[x_{1}\right]$ verifies $R=0$ and $h \in K[t]$ (instead of $h \in K\left[t, x_{2}, \ldots, x_{n}\right]$ ).

Proof. If $P$ admits a decomposition as in Theorem 1 with $R=0$ and $h \in K[t]$, then $P=h \circ Q$ is $d$-decomposable.

Conversely if $P$ is $d$-decomposable in $K\left[x_{1}, \ldots, x_{n}\right]$, then $P=h \circ Q$ with $h \in K[t]$, $Q \in K\left[x_{1}, \ldots, x_{n}\right]$. As $P$ is monic in $x_{1}$ we may suppose that $h$ is monic and $Q$ is monic in $x_{1}$. We can also suppose $\operatorname{coeff}\left(h, t^{d-1}\right)=0$. Therefore $h, Q$ and $R:=0$ verify the conditions of Theorem 1 in $A[x]$. As such a decomposition is unique, it ends the proof.

Example. Set $A=K[y]$ and let $P(x)=x^{6}+a_{1} x^{5}+a_{2} x^{4}+a_{3} x^{3}+a_{4} x^{2}+a_{5} x+a_{6}$ be a monic polynomial of degree 6 in $A[x]=K[x, y]$, with coefficients $a_{i}=a_{i}(y) \in$ $A=K[y]$. In the example of section 4 we have computed the decomposition $P=h \circ Q+R$ for $d=2$ and set $b_{1}=\frac{a_{1}}{2}, \quad b_{2}=\frac{a_{2}-b_{1}^{2}}{2}, \quad b_{3}=\frac{a_{3}-2 b_{1} b_{2}}{2}$. We found $h(t)=t^{2}+a_{6}-b_{3}^{2} \in A[t]$ and $R(x)=\left(a_{4}-2 b_{1} b_{3}-b_{2}^{2}\right) x^{2}+\left(a_{5}-2 b_{2} b_{3}\right) x \in A[x]$. By Proposition 7 above, we get that $P$ is 2-decomposable in $K[x, y]$ if and only if

$$
\left\{\begin{array}{l}
a_{6}-b_{3}^{2} \in K \\
a_{4}-2 b_{1} b_{3}-b_{2}^{2}=0 \quad \text { in } K[y], \\
a_{5}-2 b_{2} b_{3}=0 \quad \text { in } K[y] .
\end{array}\right.
$$

Each line yields a system of polynomial equations in the coefficients $a_{i j} \in K$ of $P(x, y)=\sum a_{i j} x^{i} y^{j} \in K[x, y]$. In particular the set of 2-decomposable monic polynomials of degree 6 in $K[x, y]$ is an affine algebraic variety. 


\section{REFERENCES}

[1] S.S. Abhyankar, T.T. Moh, Newton-Puiseux expansion and generalized Tschirnhausen transformation. I, II. J. Reine Angew. Math. 260 (1973), 47-83; and ibid. 261 (1973), 29-54. MR0337955 (49:2724)

[2] S.S. Abhyankar, T.T. Moh, Embeddings of the line in the plane. J. Reine Angew. Math. 276 (1975), 148-166. MR0379502 (52:407)

[3] A.F. Beardon, Composition factors of polynomials. The Chuang special issue. Complex Variables Theory Appl. 43 (2001), 225-239. MR.1820924 (2002a:12001)

[4] A. Bodin, P. Dèbes, S. Najib, Indecomposable polynomials and their spectrum. Acta Arith. 139 (2009), 79-100.

[5] J. von zur Gathen, Functional decomposition of polynomials: the tame case. J. Symb. Comp. 9 (1990), 281-299. MR1056628 (92a:12015)

[6] D. Kozen, S. Landau, Polynomial decomposition algorithms. J. Symb. Comp. 7 (1989), 445456. MR999513 (91c:13022)

[7] P.R. Lazov, A criterion for polynomial decomposition. Mat. Bilten 45 (1995), 43-52. MR:1394784 (97f:12002)

[8] P. Popescu-Pampu, Approximate roots. Valuation theory and its applications, Vol. II (Saskatoon, SK, 1999), Fields Inst. Commun., 33. Amer. Math. Soc., Providence, RI, 2003, 285-321. MR2018562 (2004k:14006)

[9] E.D. Rainville, Necessary conditions for polynomial solutions of certain Riccati equations. Amer. Math. Monthly 43 (1936), 473-476. MR.1523734

[10] A. Schinzel, Polynomials with special regard to reducibility. Encyclopedia of Mathematics and its Applications, 77. Cambridge University Press, Cambridge, 2000. MR.1770638 (2001h:11135)

Laboratoire Paul Painlevé, Mathématiques, Université de Lille 1, 59655 Villeneuve D'ASCQ, France

E-mail address: Arnaud.Bodin@math.univ-lille1.fr 